Mon. Not. R. Astron. Soc. 000, 132 () Printed 6 July $2021 \quad$ (MN LATEX style file v1.4)

\title{
The evolution of a supermassive binary caused by an accretion disc
}

\author{
P.B.Ivanov ${ }^{1,2,3 \star}$, J.C.B. Papaloizou ${ }^{1 \dagger}$ and A. G. Polnarev ${ }^{1,3 \ddagger}$ \\ ${ }^{1}$ Queen Mary \& Westfield College, University of London, London E1 4NS, United Kingdom \\ 2 Teoretical Astrophysics Center, Juliane Maries Vej 30, DK-2100 Copenhagen $\varnothing$, Denmark \\ 3 Astro Space Center of P.N. Lebedev Physical Institute of the Russian Academy of Sciences, \\ Profsoyuznaya st., 84/32, Moscow 117810, Russia
}

6 July 2021

\begin{abstract}
The interaction between a massive binary and a non-self-gravitating circumbinary accretion disc is considered. The shape of the stationary twisted disc produced by the binary is calculated. It is shown that the inner part of the disc must lie in the binary orbital plane for any value of the ivi@tfa.fy.chalmers.se viscosity.

When the inner disc midplane is aligned with the binary orbital plane on the scales of interest and it rotates in the same sense as the binary, the modification to the disc structure and the rate of decay of the binary orbit, assumed circular, due to tidal exchange of angular momentum with the disc, are calculated. It is shown that the modified disc structure is well described by a self-similar solution of the non-linear diffusion equation governing the evolution of the disc surface density. The calculated time scale for decay of the binary orbit is always smaller than "accretion" time scale $t_{a c c}=m / \dot{M}(m$ is the mass of secondary component, and $\dot{M}$ is the disc accretion rate), and is determined by ratio of secondary mass $m$, assumed to be much smaller than the primary mass, the disc mass inside the initial binary orbit, and the form of viscosity in the disc.
\end{abstract}

Key words: accretion, accretion discs-hydrodynamics-black hole physicsgalactic centers-disc satellite interactions

(c) RAS 


\section{INTRODUCTION}

In the present paper we discuss effects of interaction between a massive binary and circumbinary accretion disc. We consider in detail the evolution of the binary orbital parameters and the disc surface density.

A massive binary surrounded by a gas disc may be found in different astrophysical situations. For example, such a system may contain two pre-main-sequence stars surrounded by a protostellar disc. The tidal influence of this disc on the binary ormit may cause orbital migration, and in this way explain the observed small separations of some pre-main-sequence binaries. Similar processes may operate in protoplanetary discs producing short period planets. The influence of the binary on the disc may be to significantly modify the disc structure by opening up a gap. The effect of such a gap may may be to reduce the total luminosity of the disc, and change its spectrum, and therefore it has observational consequences (see, e.g. Jensen et al 1996; Jensen \& Mathieu 1997 for recent observations and an overview of theoretical models).

Probably an even more important example is a supermassive binary black hole which is expected to be found in the centers of some galaxies. Such a system may be formed as a result of a galaxy merging process, and the evolution may end with the coalescence of the black holes. The burst of gravitational waves generated during such a coalescence should be easily detectable by the future generation of gravitational wave antennas. Just as in the case of binary stars, the interaction of a binary black hole with an accretion disc has prominent evolutionary aspects. The disc is able to collect and radiate away orbital binding energy, and thus takes part in the process of decay of the black hole separation distance.

In fact the disc orbit interaction may determine the evolution of the binary separation distance over a definite range of scales. To see this let us briefly review the possible causes of evolution of the separation distance (Begelman, Blandford \& Rees 1980, hereafter BBR). After the merger of the galaxies, the holes sink toward the center of of the stellar distribution (central stellar cluster) due to dynamical friction. When they are within a small

\footnotetext{
* E-mail:pavel@tac.dk

$\dagger$ J.C.B.Papaloizou@qmw.ac.uk

$\ddagger$ A.G.Polnarev@qmw.ac.uk
}

(C) RAS, MNRAS 000, 132 
distance from the center $\sim 1-10 p c$ they form a gravitationally bound binary. After that the binary separation distance $r_{b}$ shrinks as a result of dynamical friction to another smaller characteristic scale $r_{\text {crit }} \sim 10^{-2}-1 p c$ where the binary becomes "hard" with respect to stellar population, i.e. the velocity obtained by a star during some typical encounter with the binary becomes larger than escape velocity of stars from the stellar cluster. Thus, a "loss cone" is formed in the velocity distribution of the stars in the cluster, and the binary can effectively interact only with stars which penetrate into "loss cone" at a rate determined by star-star relaxation processes.

For separations $r_{b} \sim 10^{-2}-1 p c$ decay of the binary orbit due to emission of gravitational waves appears to be insignificant, and the decay time scale is mainly determined by the two-body relaxation time of the central stellar cluster, which may be much larger than the cosmological time (BBR, see also Polnarev \& Rees 1994; Quinlan 1996; Quinlan \& Hernquist 1997 and references therein for a more recent discussion).

Note that the successive encounters of the stars with the binary lead also to a "random walk" of the binary center of mass around the center of stellar distribution (Bahcall \& Wolf 1976; Young 1977), and this effect may "smear out" the loss cone (Young 1977; Quinlan \& Hernquist 1997). Although the problem of speeding up the orbital evolution of the binary might be solved in such a way, it would be very plausible to suppose there is another mechanism that can produce binary orbital energy loss at "intermediate" scales $\sim 10^{-2}-1 p c$. In this connection we note that the size of the thin accretion disc around the more massive black hole (primary) may be as large as $\sim 10^{-2}-10^{-1} p c$, and therefore the interaction between the binary and the disc may "switch on" at the most critical "intermediate" scales $\sim 10^{-2}-10^{-1} p c$.

The black hole binary-disc interaction can also have significant observational consequences in its own right. As we shall see in the next Section, during the initial stage of the binary-disc interaction the orbit of the smaller (secondary) black hole can be inclined with respect to the circumprimary disc, and in this case the secondary hits the disc twice during its orbital period. At the time of secondary-disc collision outflows of hot gas from the disc are formed, and the luminosity of the outflowing gas can be of order of secondary Eddington luminosity (Ivanov et al 1998, hereafter IIN). Therefore the binary black hole may manifest itself as a periodic source of non-stationary radiation coming from the centers (C) RAS, MNRAS 000, 12 
of galaxies (see e.g. INN; Lehto \& Valtonen 1996 and references therein). As we see below, during the final stage of the binary-disc interaction the binary orbital plane and the disc plane are aligned, and as for protostellar discs, the disc structure is modified by the gap formation process. The specific spectral features expected from such a modified disc can also be used to test the hypothesis of supermassive binary black holes.

In this Paper we consider the evolutionary aspect of the interaction between a massive binary system (presumably, a binary black hole) and an accretion disc. We assume that our system consists of two companions with nonequivalent masses: a massive primary with mass $M$, and a secondary with mass $m \ll M$; it also contains an accretion disc around the primary. We also assume that in course of previous evolution the binary separation distance $r_{b}$ becomes smaller than the disc size $r_{d}$, so the disc mass inside the binary orbit $M_{d 0}\left(r_{b}\right) \leq M_{d 0}\left(r_{d}\right)$, where $M_{d 0}\left(r_{d}\right)$ is total mass of the disc (sometimes we shall call the distance $r_{b}$ the orbital distance of the secondary). We pay special attention to the case of a rather massive secondary: $M_{d 0}\left(r_{b}\right)<m \leq M_{d 0}\left(r_{d}\right)$. Such a massive secondary significantly disturbs the inner part of the disc, and causes the evolution of the disc into a disturbed quasistationary state during a relatively short time-scale. The subsequent evolution of the binary parameters takes a much longer time, and can be calculated for a given perturbed state of the disc.

In the beginning stages of the binary-disc interaction, the binary orbital plane is not necessary aligned with the disc plane, and therefore the gravitational field of the binary leads to disc distortions of two types: "horizontal" twisted distortions of the disc shape due to action of the non-spherical part of the binary gravitational potential, and "radial" distortions of the disc structure due to the tidal torque from the secondary acting on the disc gas particles which are orbiting close to the binary orbit. The "horizontal" distortions cause the formation of a quasi-stationary twisted disc where the inner part of the disc ( with radius smaller than some alignment radius $r_{a l}$ ) lies in the binary orbital plane, and the orientation of the outer part of the disc is determined by the disc formation processes (see also Section 2,3). We calculate the shape of this twisted disc, and discuss the dependence of alignment scale on viscosity and mass ratio $q=m / M$ (see also Kumar 1987,1990). The tidal torque of the secondary inhibits the disc gas from drifting inside the secondary orbit (Lin \& Papaloizou 1979 ; Goldreich \& Tremaine 1980), and so it accumulates in some region 
outside the secondary orbit, and the radial structure of the disc in that region is modified (e.g. Syer \& Clarke 1995). We calculate analytically the disc structure in that region in two ways: a) by using the laws of conservation of mass and angular momentum and b) by finding the appropriate self-similar solution of the diffusion equation which governs the viscous evolution of the surface density in the disc, and comparing the results with the results of simple numerical calculations. We also calculate the evolution of the secondary orbital separation $r_{b}(t)$ due to the torque acting between the modified part of the disc and the secondary.

Our paper is organized as follows. In the next Section we estimate the characteristic time and spatial scales relevant for interaction between the binary and the disc, and qualitatively describe the possible evolution of such a system. All parameters are assumed to be typical for a supermassive binary black hole and galactic accretion disc. In Section 3 we calculate the shape of the stationary twisted disc around an inclined binary. In Section 4 we calculate the "radial" evolution of the binary and the disc. We summarize our results in Section 5. Although we mainly discuss the case of a supermassive binary black hole, our results can be applied to other possible situations such as stellar binaries or protoplanetary discs, with minor changes. The reader who is mainly interested in twisted discs around binary systems, can go directly to Section 3, and the reader who is mainly interested in the general aspects of the "radial" evolution of the binary and the disc, can jump to Section 4.

We consider hereafter only circular binaries, and set eccentricity $e$ to zero in our calculations.

\section{MAIN PARAMETERS AND BASIC ESTIMATES}

In the beginning of this Section let us shortly review the basic properties of steady-state accretion discs. The detailed disc model is unimportant for us, and we employ simple $\alpha$-disc models (Shakura \& Sunyaev 1973) which are parameterized by the well known viscosity parameter $\alpha$ and accretion rate $\dot{M}$. For a typical AGN disc the values of viscosity and dimensionless accretion rate are assumed to be small: $\alpha \sim 10^{-2}, \dot{M} / \dot{M}_{E} \sim 10^{-2}$, where $\dot{M}_{E}$ is the Eddington accretion rate

$$
\dot{M}_{E}=\frac{4 \pi G M}{c \kappa_{T}} \approx 2 \times 10^{-1} M_{8}\left(M_{\odot} / y r\right),
$$

(C) RAS, MNRAS 000, 132 
here $\kappa_{T}$ is Thompson opacity coefficient, and $M_{8}=M / 10^{8} M_{\odot}$, and all other symbols have their usual meaning. We use later renormalized values of viscosity and accretion rate: $\alpha_{*}=$ $\alpha / 10^{-2}$, and $\dot{M}_{*}=10^{2} \dot{M} / \dot{M}_{E}$.

All interesting disc quantities can easily be expressed in terms of disc semi-thickness $h$, and steady state surface density $\Sigma_{0}$. In the gas pressure dominated case, the semi-thickness is approximately proportional to the distance $r$, and to describe the vertical extent of the disc it is convenient to introduce the disc opening angle:

$$
\delta=\frac{h}{r} \approx 10^{-3} \alpha_{*}^{-1 / 10} M_{8}^{-1 / 10} \dot{M}_{*}^{1 / 5}\left(r_{3}\right)^{1 / 20},
$$

where the distance $r_{3}=\frac{r}{10^{3} r_{g}}$ is assumed to be of order of the secondary orbital distance $r_{b}$, and gravitational radius $r_{g}=2 G M / c^{2}$. S The steady state disc surface density is given by expression

$$
\Sigma_{0} \approx 10^{5} \alpha_{*}^{-4 / 5} M_{8}^{1 / 5} \dot{M}_{*}^{3 / 5} r_{3}^{-3 / 5} \mathrm{~g} / \mathrm{cm}^{2} .
$$

With help of the expression (3) we can easily estimate the unperturbed disc mass $M_{d 0}$ inside the secondary orbit

$$
M_{d 0}\left(r_{b}\right) \approx \frac{10}{7} \pi \Sigma_{0}\left(r_{b}\right) r_{b}^{2} \sim 2 \cdot 10^{5} \alpha_{*}^{-4 / 5} M_{8}^{11 / 5} \dot{M}_{*}^{3 / 5} r_{3}^{7 / 5} M_{\odot} .
$$

The disc gas particles are orbiting around the central source with approximately Keplerian angular velocity

$$
\Omega_{K}=\sqrt{\frac{G M}{r^{3}}} \approx 0.7 \cdot M_{8}^{-1} r_{3}^{-3 / 2}(y r)^{-1},
$$

and slowly drifting toward the center due to the action of viscous forces. The characteristic "viscous" time scale follows from eqs. $(1,4)$ :

$$
t_{\nu 0}=\frac{M_{d}}{\dot{M}} \sim 10^{8} \alpha_{*}^{-4 / 5} M_{8}^{6 / 5} \dot{M}_{*}^{-2 / 5} r_{3}^{7 / 5} y r
$$

Sometimes we also use the simple estimate for the drift time scale:

$$
t_{\nu 0} \sim \alpha^{-1} \delta^{-2} \Omega_{K}^{-1}
$$

The size of a thin 'standard' disc $r_{d}$ may be determined by the specific angular momentum of the gas which enters the disc, or by processes of star formation in the outermost regions of the disc, or any processes which can 'turn off' accretion. For the "standard" accretion

$\S$ In this Section we assume that opacity law in the disc is determined by Thompson opacity. The expressions for other reasonable opacity laws give similar results (see eqs. (44-47) for a general case).

(C) RAS, MNRAS 000, 132 
disc with value of viscosity parameter $\alpha \sim 10^{-2}$, the Toomre stability parameter $Q$ can be rather small:

$$
Q \approx \frac{\Omega_{K} c_{s}}{\pi G \Sigma} \approx \frac{\delta M}{M_{d 0}} \approx 0.5 \alpha_{*}^{7 / 10} M_{8}^{-13 / 10} \dot{M}_{*}^{-2 / 5} r_{3}^{-27 / 20}
$$

In the disc region where $Q<1$ the disc is gravitationally unstable and locally gas may collapse to form low-mass stars (e. g. Spitzer \& Saslaw 1966, Illarionov \& Romanova 1988). We do not consider such a disc here as we adopt disc parameters such that $Q \geq 1$. As mentioned above, we consider the case when the interaction between the secondary and the disc occurs on scales smaller than the disc size $r_{d}$. That condition can be described in terms of characteristic radial scale $r_{m}$-the scale where the disc mass is equal to the mass of the secondary:

$$
r_{m} \approx 3 \cdot 10^{3} \alpha_{*}^{4 / 7} m_{6}^{5 / 7} M_{8}^{-11 / 7} \dot{M}_{*}^{-3 / 7} r_{g}
$$

where $m_{6}=m / 10^{6} M_{\odot}$. The secondary interacts only with gas contained inside the scale $r_{m}$, and the disc region outside this radius is in the unperturbed steady state $\square$. The gas contained inside the radius $r_{m}$ is accreted during the time $t_{a c c}=\frac{m}{\dot{M}}$ :

$$
t_{a c c}=t_{\nu 0}\left(r_{m}\right)=5 \cdot 10^{8} \frac{m_{6}}{M_{8}} \dot{M}_{*}^{-1} y r
$$

and this time is larger than any characteristic time scale relevant to our problem. In the case of a supermassive binary black hole, the 'accretion' time (8) must be smaller than cosmological time in order to provide the source of gravitational radiation (BBR).

Now let us describe qualitatively the scenario for binary evolution due to the interaction with the accretion disc, and estimate the relevant characteristic spatial and time scales. As we will see the interaction of the secondary with the disc gas leads to alignment of the secondary orbital plane and the plane of the inner disc. After the disc alignment has occurred, the orbital separation starts to shrink in the radial direction. Therefore the evolution of the binary and disc can be roughly divided into two successive stages -the alignment stage and the stage of 'radial' evolution, and we consider these stages in turn.

First we assume that the secondary orbital plane is inclined with respect to the disc plane. The effects of the interaction between the inclined secondary and the disc can be

I Actually, in case of a very massive secondary, the disc structure is close to the steady state solution at scales smaller than $r_{m}$, see eqs. $(63,64)$ below.

(C) RAS, MNRAS 000, 132 
considered in two regimes. The first is when highly non-stationary distortions of the disc result from direct collisions between the secondary and disc (s.-d. collisions). The second is when the long-ranged, averaged over many orbital periods, interaction between the binary and the disc produces a twisted warped disc.

As we will see the evolution of the secondary orbit due to s.-d. collisions is important for relatively low-mass secondaries, and long-ranged interaction is important in the opposite case.

Let us estimate effects of the direct collisions between the secondary and the disc. The inclined secondary hits the disc twice during an orbital period $t_{\text {orb }}$, which is approximately equal to the Keplerian period:

$$
t_{\text {orb }} \sim 2 \pi \Omega_{K}^{-1}
$$

During a collision, the secondary significantly perturbs the disc gas at distances from it comparable with the "accretion" radius:

$$
r_{a}=\frac{2 G m}{\left|\vec{v}_{s e c}-\vec{v}_{d}\right|^{2}} \approx 3 \times 10^{14} m_{6} r_{3} c m
$$

where $\vec{v}_{\text {sec }}$ is the velocity of the secondary which intersects the disc at the "collision" radius $r_{c} \sim r_{b}, \vec{v}_{d}$ is the disc velocity at the point of collision, and $\left|\vec{v}_{s e c}\right| \sim\left|\vec{v}_{d}\right| \sim r_{c} \Omega\left(r_{c}\right)$. The perturbed gas is heated up by the shock induced in the disc. An amount of gas of mass $\sim \pi \Sigma r_{a}^{2}$ is accreted by the secondary, another amount of approximately the same mass attains velocities greater than the escape velocity in the binary potential and leaves the system (IIN). As result of the collision, the linear momentum of the secondary is changed, with the momentum change $\delta \vec{p}_{s e c}$ being proportional to the total mass of perturbed region $>2 \pi \Sigma\left(r_{b}\right) r_{a}^{2}$, and it is directed opposite to the relative velocity of the secondary $\vec{v}_{s e c}-\vec{v}_{d}$. Therefore the change of velocity of the secondary $\delta \vec{v}_{\text {sec }}$ can be written as:

$$
\delta \vec{v}_{s e c} \approx-\frac{2 \pi \sum r_{a}^{2} A}{m}\left(\vec{v}_{s e c}-\vec{v}_{d}\right)
$$

here the correction factor $A>1$ is mainly determined by the disc distortions at scales larger than $r_{a}$ 灲. The secondary orbital parameters change on account of the change of velocity, and this leads to the dragging of the secondary orbit into the disc plane. To see this, consider

$\|$ We do not take into account the resonant excitation of waves in the disc due to the secondary passing through the disc. Estimates of this effect have been made e. g. by Artymowicz 1994.

(C) RAS, MNRAS 000, 132 
the evolution of the inclination angle $\beta$ : the angle between the specific angular momentum of the secondary $\vec{l}_{s e c}$, and the specific angular momentum vector of the disc gas $\vec{l}_{d}=\vec{r}_{c} \times \vec{v}_{d}$ (see Fig. 1 below). From eq. (11) it is easy to obtain the equation for the change of this angle during the collision

$$
\delta \beta=-\frac{2 \pi \Sigma r_{a}^{2} A}{m} \frac{\left|\vec{l}_{d}\right|}{\left|\vec{l}_{s e c}\right|} \sin (\beta) .
$$

One can easily see from this expression that there is only one stable value of $\beta$ : $\beta=0$. Thus, collisions between the secondary and the disc tend to align the angular momentum vectors of the disc and the secondary. When these collisions determine the evolution of the secondary orbital parameters, the secondary is progressively dragged into the disc plane until it is in a coplanar orbit. The associated timescale $t_{\text {dragg }}$ can be easily estimated with help of eq. (11) to be

$$
t_{\text {drag }} \approx \frac{m}{2 \pi \Sigma r_{a}^{2} A} t_{\text {orb }}
$$

As we mentioned above the b.h-disc collisions result in an outflow of disc gas at a rate

$$
\dot{M}_{\text {out }}>\Sigma_{0} r_{a}^{2} \Omega_{K}
$$

Obviously, this outflow must be compensated by a radial inflow of mass through the disc, and therefore the surface density in eqs. (11-13) is close to the steady state value (3) only if the condition $\dot{M}_{\text {out }}<\dot{M}$ is satisfied. This condition gives:

$$
q<\sqrt{\frac{3 \pi}{4}} \alpha^{1 / 2} \delta \sim 1.5 \cdot 10^{-4} B \alpha_{*}^{1 / 2},
$$

where black holes mass ratio

$$
q=\frac{m}{M}
$$

the parameter $B=\delta / 10^{-3} \approx 1$, and we use the standard equation of stationary disc accretion: $\dot{M}=3 \pi \nu \Sigma_{0}$ and kinematic viscosity $\nu=\alpha \delta^{2} r^{2} \Omega_{K}$. In the opposite case $q>\alpha_{*}^{1 / 2} \delta$, the secondary depletes gas from the region of the disc near its orbit, so the surface density in this region should be much less than the unperturbed surface density $\Sigma_{0}$. Now an upper limit for the surface density $\Sigma\left(r_{c}\right)$ may be estimated by equating the rate of outflow of disc

\footnotetext{
$\star \star$ In the analogous case of star-disc collision, similar calculations and references to earlier work can be found in a paper by Vokrouhlicky \& Karas 1998.
}

(C) RAS, MNRAS 000, 132 
gas $\sim \Sigma\left(r_{c}\right) r_{a}^{2} \Omega_{K}$, and the disc accretion rate $\dot{M}$, and that gives

$$
\Sigma\left(r_{p}\right) \sim \frac{\alpha \delta^{2}}{q^{2}} \Sigma_{0} \ll \Sigma_{0}
$$

In the limit $\frac{\alpha \delta^{2}}{q^{2}} \ll 1$ the drag timescale is of order of 'accretion' time scale $t_{a c c}$.

In the case of a sufficiently massive binary, the disc midplane is expected to be aligned with the binary orbital plane due to the influence of quadrupole component of the binary gravitational field. Schematically this process can be divided again into two successive stages. The planar disc first evolves into a quasi-stationary twisted configuration (the twisted disc) during some characteristic alignment time $t_{a l}$. In the twisted disc, the inner part of the disc is aligned with the binary orbital plane, and the outer part does not change its orientation. The radius out to which the disc is aligned $\left(r_{a l}\right)$, as well as the alignment time $t_{a l}$, are estimated in the next Section (see also Kumar 1987, 1990). Both quantities strongly depend on the value of viscosity parameter $\alpha$. For sufficiently large values of $\alpha>\delta$, we obtain:

$$
r_{a l 1} \sim(\alpha q)^{1 / 2} \delta^{-1} r_{b}
$$

and diffusion-like decay to a quasi-stationary twisted disc proceeds during the time

$$
t_{a l} \sim \alpha^{2} t_{\nu 0} \sim \alpha \delta^{-2} \Omega_{K}^{-1}
$$

For small values of $\alpha$, the alignment scale does not depend on viscosity (Ivanov \& Illarionov 1997, hereafter II)

$$
r_{a l 2} \sim q^{1 / 2} \delta^{-1 / 2} r_{b}
$$

and the evolution to the stationary configuration has wave-like character (Papaloizou \& Lin 1995, hereafter PL). Now the alignment time $t_{a l}$ is approximately equal to the 'sound' time $t_{s}$ : the time taken by sound wave to cross the radius $r$ :

$$
t_{s} \sim \delta^{-1} \Omega_{K}^{-1}\left(r_{a l}\right) .
$$

Obviously, the alignment scale must be greater than the binary orbital distance: $\max \left(r_{a l 1}, r_{a l 2}\right)>$ $r_{b}$. That condition gives:

$$
q>\frac{\delta^{2}}{\alpha} \quad \text { when } \quad \alpha>\delta ; \quad q>\delta \text { when } \alpha<\delta .
$$

When the inequalities (21) are broken, the alignment effect is absent.

After the formation of a quasistationary twisted disc, the orientation of the binary orbital plane itself slowly changes with time due to the influence of the gas accreting through the twisted disc. When the gas is accreting through the twisted disc, the orientation of its angular

(C) RAS, MNRAS 000, 132 
momentum is changing, and therefore the orientation of the binary orbital plane must also be change with time due to the law of conservation of angular momentum (e. g. Rees 1978). This fact allows us to estimate the change with time of the binary inclination angle $\beta_{\text {out }}$ as:

$$
\dot{\beta}_{\text {out }} \sim-\frac{\beta_{\text {out }}}{t_{*}}, \quad \beta_{\text {out }}(t) \propto e^{-\frac{t}{t_{*}}}
$$

where index out means that the inclination is determined with respect to the orientation of the disc at radii far from the binary. The characteristic time-scale of this effect $t_{*}$ is determined by the ratio of specific angular momentum of the binary to the specific angular momentum of the disc at radius $r \sim r_{a l}$, and by the 'accretion' time $t_{a c c}$ :

$$
t_{*} \sim \frac{r_{b} v_{d}\left(r_{b}\right)}{r_{a l} v_{d}\left(r_{a l}\right)} t_{a c c}
$$

Here the alignment scale is given by eq. (17) and $\alpha$ is assumed to be rather large $(\alpha \sim 1)$ (the case of small $\alpha \ll 1$ is more complicated and must be considered separately, see e.g. Scheuer \& Feiler 1996). When the secondary counter rotates with respect to the disc gas ( retrograde rotation), we have $\frac{v_{b}}{v_{d}}<0$, and the time $t_{e v}$ is formally negative. This means that the inclination of the binary orbital plane with respect to the disc plane grows with time, and the binary may turn over and become prograde during the time $\sim t_{e v}$. Note, however that a highly inclined twisted disc may be unstable with respect to instabilities of a different kind, and so the evolution of a retrograde inclined binary is unclear. We will assume below that the binary is rotating prograde with respect to the disc gas.

From eq. $(23)$ and eqs. $(18,20)$ it follows that $t_{e v} \gg t_{a l}$ when $m \gg M_{d}$. This inequality as well as the inequalities (21) tell us that the disc alignment effects are important only for sufficiently massive secondaries (typically $q>10^{-3}$ ). In the opposite case the secondary is quickly dragged into the disc midplane by the competing effects of secondary-disc collisions.

Now let us assume that the binary and the disc are aligned at scales of interest, and consider the next 'radial' stage of the evolution of the binary and the disc. At first let us look at the case of the small mass of the secondary $m<M_{d}$. As we mentioned above a low massive secondary must lie in the disc plane corotating with the disc gas. Orbiting in the disc the secondary transfers the angular momentum to the neighboring gas particles which are orbiting upstream, and gain the angular momentum from downstream gas particles due to resonance interactions/ non-resonance scattering of these particles (Lin \& Papaloizou 1979 Goldreich \& Treimain 1980). This can lead to the formation of gap around the secondary 
which is free from the gas, provided the gap formation criterion is satisfied (see, e.g. Lin \& Papaloizou 1979, 1986)

$$
q>\frac{81 \pi \nu}{8 r_{b}^{2} \Omega_{K}}
$$

where $r_{b}$ is the binary separation distance. For $\alpha$-discs the criterion (24) can be rewritten as

$$
q>\frac{81 \pi}{8} \alpha \delta^{2} \sim 3 \times 10^{-7} \alpha_{*}
$$

One can see from this relation that the criterion is obviously satisfied for any reasonable mass of the secondary. Lin and Papaloizou showed that when the gap is opened the orbit of secondary is shrinking during viscous time scale $t_{\nu 0}$ i.

At the end of this section let us briefly discuss the second possibility $m>M_{d}$ ( this case is discussed in detail in Section 3). Now the radial drift of the secondary proceeds during the time much larger than $t_{\nu 0}$. The secondary torque acting on the downstream gas particle prevents them to drift inside the secondary orbit, and the downstream gas is accumulated in some region outside the secondary orbit. The structure of the disc in that region is significantly modified. The radius out to which the disc is modified grows with time (see Section 3). The accumulated mass of the gas near the secondary orbit increases the torque acting on the secondary, and pushes the secondary toward the primary. The secondary orbit is shrinking during the characteristic evolution time-scale $t_{e v}\left(t_{\nu 0} \ll t_{e v}<t_{a c c}\right)$, which is evaluated in the Section 3.

\section{THE TWISTED ACCRETION DISC AROUND THE INCLINED BINARY}

Now let us consider in more detail the evolution and the structure of the twisted disc around the binary. At first we need to choose a convenient coordinate system. As a basic coordinate system we use the Cartesian coordinate system $(x, y, z)$, which has its origin $(\mathrm{O})$ at the center of mass of the binary. The plane (XOY) coincides with the binary orbital plane and the axis (OZ) is directed perpendicular to that plane. As it follows from our previous discussion the binary orbital plane is slowly evolving with time, but when considering the dynamics and

†† Note that the secondary can shrink its orbit even though it is not massive enough to open a gap (Ward 1997 and references therein)

(C) RAS, MNRAS 000, 132 


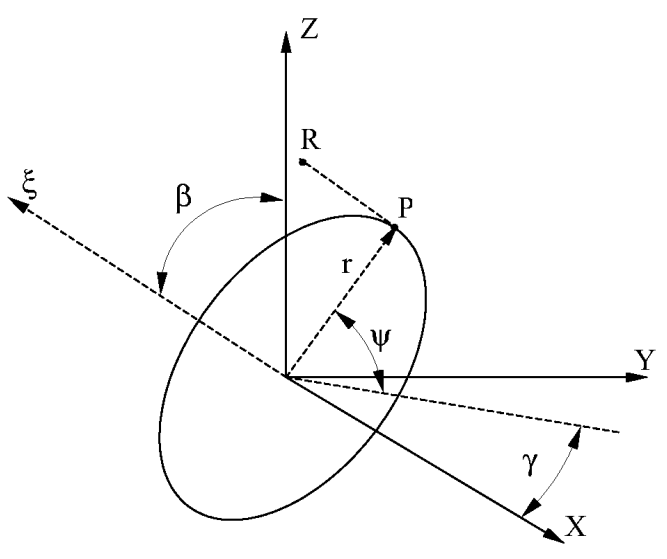

Figure 1. The $r, \psi, \xi$ coordinate system shown with respect to the Cartesian coordinate system. $P$ is the projection of the point $R$ on to the disc ring plane.

the structure of the twisted disc itself the effect of this evolution is unimportant. Since the twisted disc has some inclination with respect to the binary plane, and this inclination is different for different radii $r$, when studying the local properties of the disc it is convenient to use another locally cylindrical (twisted) coordinate system $(r, \psi, \xi)$, (Petterson 1977). This system consists of rings centered at the point $(\mathrm{O})$, which have different (with radius) inclination angles $\beta(r, t)$ with respect to the binary orbital plane, and the lines of nodes of these rings are rotated through some angle $\gamma(r, t)$ with respect to the (OX)-axis, see Fig. 1. The position of the gas elements on the ring is characterized by the angle $\psi$, and the coordinate $\xi$ characterizes the vertical displacement of the gas element out of the ring plane (see Fig. 1). Assuming that the twisted disc is evolving on a time scale much larger than the orbital time scale, and the spatial scales of the disc twist are much larger than the binary orbital distance, we hereafter use the time-averaged over many orbital periods monopole and quadrupole components of the binary potential $\phi(r, \psi, \xi)$ :

$$
\phi(r, \psi, \xi)=-\frac{G M}{R}-\frac{G m r_{b}^{2}}{2 r^{3}}\left(\frac{3}{2} \frac{R^{2}}{r^{2}}\left(1-\frac{2 \xi \beta \sin \psi}{R}\right)-1\right),
$$

where the spherical coordinate $R=\sqrt{r^{2}+\xi^{2}}$, and we assume $\beta<1$ 胡.

When disc twist is small $(\beta \ll 1)$, trajectories of the gas particles in the twisted disc are

$\ddagger \ddagger$ In the expression (25) the assumption $m \ll M$ can be relaxed. The potential (25) describes the time-averaged gravitational field of the circular binary with components having the comparable masses $m_{1}, m_{2}$, provided $M=m_{1}+m_{2}$, and $m=\frac{m_{1} m_{2}}{m_{1}+m_{2}}$. 
ellipses with small eccentricity $e$. The influence of the non-spherical part of the potential (25) on these trajectories can be described as the following. Neglecting the interaction between gas particles and then considering the free motion of these particles in the potential (25), one can see that the quadrupole contribution to the potential causes the precession of the major axis of an elliptical orbit with the frequency:

$$
\Omega_{a p}=\frac{3}{4} q\left(\frac{r_{b}}{r}\right)^{2} \Omega_{K}
$$

and precession of the lines of nodes with the frequency:

$$
\Omega_{n p}=-\Omega_{a p}
$$

The apsidal precession is prograde and the nodal precession is retrograde with respect to the orbital motion. As we will see, the signs of these precession frequencies determines the shape of the stationary twisted disc in the low-viscosity limit.

The dynamics and the structure of a twisted disc can be described in terms of two complex variables: $\mathbf{W}(r, t)=\beta e^{i \gamma}$, and $\mathbf{A}(r, t)$. The variable $\mathbf{W}$ determines the inclination

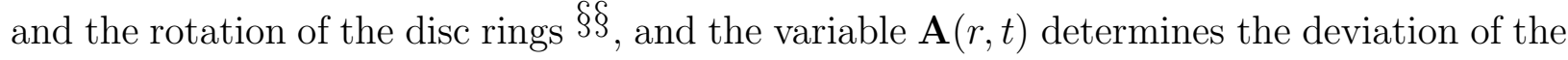
gas trajectories from the circle of the radius $r$. Namely, the eccentricity $e$ and the position of the apsidal line of the elliptical trajectory $\psi_{0}$ can be expressed in terms of $\mathbf{A}: e=-2 \frac{\xi}{r} \Omega_{K}|\mathbf{A}|$, $\psi_{0}=\arccos \frac{R e \mathbf{A}}{|\mathbf{A}|}$ (Demianski \& Ivanov 1997). In the low-viscosity limit $\alpha<1$, the dynamical twist equations can be written as

$$
\begin{gathered}
\dot{\mathbf{A}}=-\frac{G M}{4 r^{2}} \mathbf{W}_{, r}+\left(i \Omega_{a p}-\alpha \Omega_{K}\right) \mathbf{A}, \\
\dot{\mathbf{W}}=-\frac{\delta^{2}}{r} \frac{\partial}{\partial r}\left(r^{2} \mathbf{A}\right)+i \Omega_{n p} \mathbf{W} .
\end{gathered}
$$

The derivation of these equations lies beyond the scope of our paper, but can be found in papers by Papaloizou and Pringle 1983, Papaloizou and Lin 1995, Ivanov and Illarionov 1997, Demianski and Ivanov 1997. Note that the equations (28-29) are applicable not only to the case of a binary system, but also for general perturbing forces which can cause apsidal and nodal precession of the disc gas trajectories in the linear approximation over the angle $\beta$ (II, Demianski \& Ivanov 1997).

We do not solve the time dependent form of these equations in our work, and restrict $\S \S$ The variable $\mathbf{W}$ is connected to the variable $g$ used by PL by $\mathbf{W}=(i g)^{*}$. 
ourselves to qualitative analysis only 땡. For simplicity in our analysis of the time dependent case we also neglect the terms proportional to the precession frequencies -the last terms on the right hand side of eqs. (28), (29) (for more detailed analysis see, e.g Papaloizou \& Lin 1997). These terms determine the shape of the stationary solutions of the twist equations, but are not important for the character of time behavior. Let us also look for the solution of eqs. (28), (29) in the WKB-limit: $\mathbf{W} \propto e^{i(\omega(k) t-k r)}, k r<1$. We obtain the dispersion relation $\omega(k)$ in the form $(\mathrm{PL})$ :

$$
\omega=\frac{1}{2}\left(i \alpha \Omega_{K} \pm \sqrt{k^{2} v_{s}^{2}-\alpha^{2} \Omega_{K}^{2}}\right)
$$

where $v_{s}=\delta r \Omega_{K}$ is the sound velocity. In the case $k>\frac{\alpha \Omega_{K}}{v_{s}}$ eq. (30) describes the propagation of the waves with velocity $v_{W} \approx v_{s} / 2$. In the opposite case $k<\frac{\alpha \Omega_{K}}{v_{s}}$ the mode corresponding to the sign $(+)$ in the eq. (28) describes the decay of the ellipticity of the gas trajectories due to the action of viscous forces. The mode corresponding to the sign $(-)$ is described by the relation

$$
\omega \approx i \frac{\delta^{2}}{4 \alpha}\left(r^{2} k^{2}\right) \Omega_{K}
$$

This relation implies that the relaxation to the stationary configuration has diffusion-like character, and the characteristic time scale of this effect $t_{a l}(k) \sim \alpha \delta^{-2}(r k)^{-2} t_{\text {orb }}$. Considering the modes with $k \sim r$, it is easy to see that the transition from wave-like to diffusion-like propagation of the twist corresponds to $\alpha>\delta$, and when $\alpha>\delta$ the disc alignment time-scale $t_{a l}$ can be estimated as:

$$
t_{a l} \sim t_{a l}\left(k \sim r^{-1}\right) \sim \alpha \delta^{-2} t_{o r b}
$$

In the opposite case $\alpha<\delta$, we have:

$$
t_{a l} \sim r / v_{s} \sim \delta^{-1} t_{o r b}
$$

In stationary case eqs. $(28,29)$ can be written as:

$$
\begin{gathered}
\frac{d}{d x} \frac{x^{3 / 2}}{A} \frac{d}{d x} \mathbf{W}-\frac{3 i \tilde{q}}{x^{5 / 2}} \mathbf{W}=0, \\
A=\tilde{\alpha}-\frac{3 i}{4} \tilde{q} x^{-2}
\end{gathered}
$$

where we use the explicit form for the precession frequencies $(28,29)$, introduce the dimen-

ฯ For the discussion of numerical solutions of these equations see Kumar 1990, PL. 
sionless distance $x=\frac{r}{r_{b}}$, and new parameters $\tilde{\alpha}=\frac{\alpha}{\delta}$ and $\tilde{q}=\frac{q}{\delta}$. The eq. (32) is similar to the equation describing a stationary twisted disc around a Kerr black hole (II) except for the $(-)$ sign in front of the forcing term (the last term in (32)). Ivanov and Illarionov found radial oscillations of the inclination angle of a low-viscosity stationary twisted disc around a Kerr black hole, and suggested that this effect holds for any nearly Keplerian forces which cause apsidal and nodal precession of the disc trajectories provided that these precessions are in the same direction. $\left(\frac{\Omega_{n p}}{\Omega_{a p}}>0\right)$. They also suggested that when the precessions counter-rotated $\left(\frac{\Omega_{n p}}{\Omega_{a p}}<0\right)$ the disc inclination must join smoothly to the orbital plane of the object. A twisted disc around a binary system gives a natural example of counter-rotating precessions, and we see below in our case that the disc lies in the orbital plane for any value of viscosity.

Equation (32) cannot be solved analytically; its numerical solution is presented at Figs. 2, 3. However, we can obtain approximate analytic solutions of this equation for the case $\tilde{\alpha} \gg 1$, and $\tilde{\alpha} \ll 1$. When $\tilde{\alpha} \gg 1$ we can set $A \approx \tilde{\alpha}$, and the approximate solution of eq. (32) is expressed in terms of Kelvin functions $\operatorname{ker}(x), \operatorname{kei}(x)$ :

$$
\mathbf{W}=\frac{2^{3 / 4} \mathbf{W}_{\text {out }}}{\Gamma\left(\frac{1}{4}\right)} y_{1}^{\frac{1}{4}}\left(\operatorname{ker}_{\frac{1}{4}}\left(y_{1}\right)+\text { ikei }_{\frac{1}{4}}\left(y_{1}\right)\right),
$$

where $\Gamma(x)$ is the gamma-function, $\mathbf{W}_{\text {out }}=\mathbf{W}(r \rightarrow \infty), y_{1}=\frac{r_{a l 1}}{r}$, and

$$
r_{a l 1}=(3 \tilde{q} \tilde{\alpha})^{1 / 2} r_{b}
$$

Hereafter we assume $\mathbf{W}(r \rightarrow 0) \rightarrow 0$ as an inner boundary condition. When $r \rightarrow 0$,

$$
\mathbf{W} \approx C_{1}(r) e^{-\frac{r_{a l 1}}{\sqrt{2} r}} e^{-i\left(\frac{r_{a l 1}}{\sqrt{2} r}+\frac{3 \pi}{16}-\gamma_{o u t}\right)},
$$

where $C_{1}(r)$ is a slowly changing real function. When $\tilde{\alpha} \ll 1, A \approx-\frac{3}{4} i \tilde{q} x^{-2}$, and the solution is expressed in terms of modified Bessel function $K(x)$ :

$$
\mathbf{W}=2^{3 / 8} \frac{\mathbf{W}(\text { out })}{\Gamma(5 / 8)} y_{2}^{5 / 8} K_{5 / 8}\left(y_{2}\right)
$$

where $y_{2}=\left(\frac{r_{a l 2}}{r}\right)^{2}$, and

$$
r_{a l 2}=\frac{(3 \tilde{q})^{1 / 2}}{2} r_{b}
$$

When $r \rightarrow 0$,

$$
\mathbf{W} \propto e^{-\left(\frac{r_{a l 2}}{r}\right)^{2}}
$$

Comparing eqs (35), (38) we see that in the case of a relatively high viscosity, the disc rings

(C) RAS, MNRAS 000, 132 


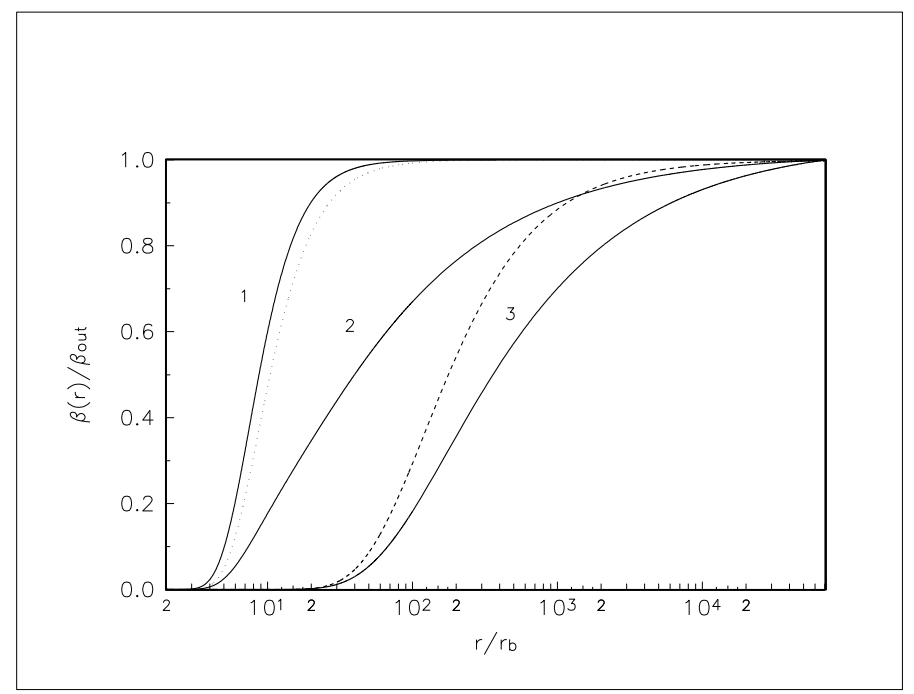

Figure 2. The dependence of the inclination angle $\beta(r) / \beta_{\text {out }}$ on radius $r / r_{b}$ for $\tilde{q}=100$, and for the different values of $\tilde{\alpha}$. Curves 1, 2 and 3 correspond to $\tilde{\alpha}=0.01,1,100$ respectively. We also plot two simple analytic approximations for these curves which correspond to small and large values of $\tilde{\alpha}$. The dotted curve $\beta=e^{-\left(\frac{r_{a l 2}}{r}\right)^{2}}\left(r_{a l 2}=8.7 r_{b}\right)$, and the dashed curve $\beta=e^{-\left(\frac{r_{a l 1}}{\sqrt{2} r}\right)}\left(r_{a l 2}(\tilde{\alpha}=100)=173.2 r_{b}\right)$ are the approximate solutions of the twist equation in the limit of high viscosity (see eqs. 35,38$)$.

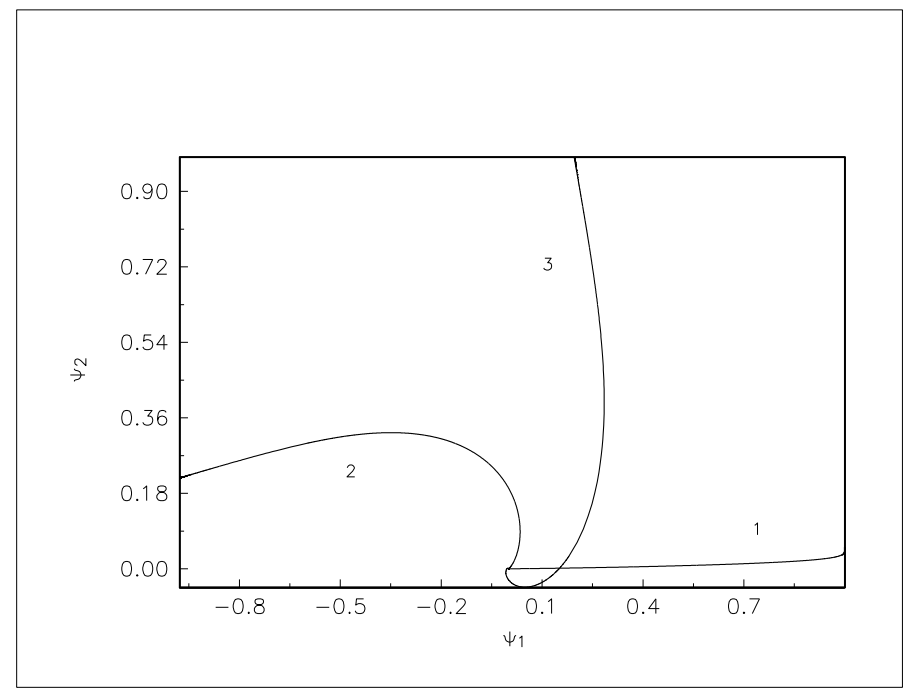

Figure 3. The result of the integration of the twist equation (32) in a parametric form $\left(\Psi_{1}=\operatorname{Re} \mathbf{W}, \Psi_{2}=\operatorname{Im} \mathbf{W}\right)$. The larger values of $\beta=\sqrt{\Psi_{1}^{2}+\Psi_{2}^{2}}$ correspond to the larger values of radial distance. The curve 1 correspond to the curve 1 of Fig. 2 $(\tilde{q}=100$ hereafter and $\tilde{\alpha}=0.01)$, the curves 2 and 3 correspond to the curves 2,3 of Fig. $2(\tilde{\alpha}=1$ and $\tilde{\alpha}=100$ respectively). When viscosity is small, the rotational angle $\gamma=\arctan \left(\frac{\Psi_{1}}{\Psi_{2}}\right)$ is not changed with the change of $r$, for larger values of viscosity the rotational angle is changing with the distance.

are twisting with $r$, but in the low viscosity limit the effect is absent. This might give a possibility of testing the value of viscosity in circumbinary twisted discs.

The general case $\tilde{\alpha} \sim 1$ must be analyzed numerically. We plot the results of numerical integration of the twist equation (32) in Figs. 2,3. 
As follows from Fig. 2., the alignment scale decreases with decrease of the viscosity parameter in agreement with our analytic estimates, and the expressions (33), (36) give a good approximation to the numerical solutions of eq. (30). Fig. 3 illustrates the change of the twist angle $\gamma$ with the distance $r$. One can see from this Figure that the angle $\gamma$ does not change significantly for small values of the viscosity parameter.

In deriving eq. (32) we have neglected the self-gravity of the disc. The effect of selfgravity is manifest in two different ways: it gives an additional non-local forcing term $F_{s g}$ in eq. (32), as well as a contribution to the resonant denominator $A$. The term $F_{s g}$ must be compared with the term induced by pressure $-F_{p}$ - the first term on left-hand side of eq. (32). According to PL, the forcing term $F_{s g}$ can be estimated as: $F_{s g} \sim \frac{\alpha}{\delta Q} F_{p}$, and we can neglect this term assuming that viscosity takes a rather small value $\|$ :

$$
\alpha<\delta Q
$$

On the other hand, the correction to the resonant denominator is more important in case of low viscosity, and can be estimated formally by setting $\alpha=0$. As well as giving a correction to the Newtonian potential due to the secondary, self-gravity induces apsidal precession of

elliptical trajectories with a frequency $\Omega_{s g} \sim \frac{M_{d}}{M} \Omega_{K}$. This frequency must be compared to the frequency $\Omega_{a p}$ at characteristic scale $r_{a l 2}$ :

$$
\Omega_{s g} \sim \frac{M_{d}\left(r_{a l 2}\right)}{M} \Omega_{K} \sim \frac{\delta}{Q} \Omega_{K}<\Omega_{a p}\left(r_{a l 2}\right) \sim \delta \Omega_{K}
$$

Thus, for estimating precession frequencies, the correction due to self-gravity appears to be less important than the correction due to gravitational field of the secondary if $Q>1$.

At the end of this Section we note that the low viscosity $\tilde{\alpha}<1$ twisted disc may be unstable with respect to shear instabilities if the "initial" inclination angle $\beta_{\text {out }}$ is sufficiently large $\beta_{\text {out }}>\delta$ (Coleman \& Kumar 1993, II). It was suggested (II) that the shear instability might result in development of turbulence and so effectively increase the value of the viscosity parameter. In this case the effective scale of the disc alignment increases and equation (37) gives only a lower limit to the alignment scale.

||| In a similar context a self-gravitating twisted disc with $(Q \sim 1)$ has been recently discussed by Papaloizou, Terquem and Lin 1998.

(C) RAS, MNRAS 000, 132 


\section{BINARY ORBIT EVOLUTION IN THE PLANE OF THE ALIGNED DISC}

Consider now in detail the radial evolution of the disc and the secondary. In this Section we assume that the disc plane is aligned with the orbital plane of the binary near the secondary orbit, and the secondary rotates in the same sense as the disc. Hereafter we adopt the following assumptions: a) we assume that the mass of the secondary is much smaller than the primary mass $M$, but larger than the characteristic disc mass $M_{d}$ b) the evolution due to interaction of the secondary and disc is switched on at some moment of time $t=t_{0}$ when the secondary lies in the disc at some radius $r_{0} \sim r_{c}<r_{d}$. The disc is taken to have a steady state structure at this moment c) Assuming that the inner (with respect to the secondary orbit) part of the disc is relatively quickly swallowed up by the primary, we do not consider the evolution of that part. d) We assume the coefficient of kinematic viscosity $\nu$ to be a power-law function of cylindrical coordinate $r$ and surface density $\Sigma$ :

$$
\nu=k \Sigma^{a} r^{b}
$$

The expression (39) can be specified for a given model of the accretion disc. In the $\alpha$-disc model the relation between surface density and viscosity follows from consideration of the energy balance. Assuming the power-law dependence of the opacity $\kappa$ on the density and temperature

$$
\kappa=\kappa_{0} \rho^{\mu_{1}} T^{\mu_{2}}
$$

a simple calculation gives (Lyubarskij \& Shakura 1987)

$$
k=f\left\{\alpha^{8+\mu_{1}-2 \mu_{2}} \kappa_{0}^{2} \mathcal{R}^{2\left(4-\mu_{2}\right)}(G M)^{\left(2 \mu_{2}+\mu_{1}-4\right) / 2}\left(c a_{r}\right)^{-2}\right\}^{\frac{1}{\epsilon}},
$$

and

$$
a=\frac{2\left(2+\mu_{1}\right)}{\epsilon}, \quad b=\frac{3\left(4-\mu_{1}-2 \mu_{2}\right)}{2 \epsilon},
$$

and $\epsilon=6-2 \mu_{2}+\mu_{1}$. Here $a_{r}$ is the radiation density constant, $\mathcal{R}$ is the gas constant, $c$ is the speed of light, and the standard system of units is assumed. The parameter $f \sim 1$ depends on the disc structure in vertical direction and is unimportant for us. If Thompson opacity dominates, $\kappa=\kappa_{T h}=0.4\left(\mathrm{~cm}^{2} \mathrm{~g}^{-1}\right), a=2 / 3$, and $b=1$. If the opacity is determined by free-free processes, $\kappa_{f f} \approx 0.8 \cdot 10^{23} \rho T^{-7 / 2}\left(\mathrm{~cm}^{2} \mathrm{~g}^{-1}\right)$, and then $a=3 / 7, b=15 / 14$.

An analogous problem was formulated and discussed by Syer and Clarke 1995 (hereafter

(C) RAS, MNRAS 000, 12 
SC). However, our results differ from those obtained by SC. As we see below the effect of the interaction between the secondary and the disc leads to accumulation of the disc gas in the region of the disc confined between the binary orbital radius $r_{b}$, and some 'binary influence' radius $r_{i n f l}(t)>r_{b}$. The radius $r_{i n f l}$ grows with time. Syer and Clarke approximated $r_{i n f l}$ as a constant, and this led to an overestimation of the disc surface density near the secondary orbit, and underestimation of the binary evolution time-scale in their calculations.

\subsection{Evolution of the disc and the binary}

The master equation for the evolution of the disc surface density $\Sigma$ can be written as (e.g Lightman \& Eardley 1974, Lyubarskii \& Shakura 1987):

$$
\frac{\partial}{\partial t} \Sigma=\frac{3 k}{r} \frac{\partial}{\partial r}\left(r^{1 / 2} \frac{\partial}{\partial r}\left(r^{1 / 2+b} \Sigma^{1+a}\right)\right) .
$$

Strictly speaking this equation is not valid near the secondary orbit, where the tidal interaction exchanges the angular momentum between the disc and the secondary. We assume hereafter that the tidal torque is concentrated in the narrow disc ring near the secondary orbit, and eq. (41) is valid at all distances outside this ring. Following this assumption the influence of the secondary on the disc can be approximated as an effective boundary condition for eq. (41), which takes into account the exchange of the $z$-component of the angular momentum between the secondary and the disc (SC)

$$
\left\{2 \pi r^{2} \Sigma\left(\frac{3}{2} \nu \Omega_{K}+v_{d r} r \Omega_{K}\right)+\frac{1}{2} m r \Omega_{K} \frac{d r_{b}}{d t}\right\}_{r=r_{b}}=0
$$

where all quantities are taken at $r=r_{b}(t)$, and the secondary radial velocity $\frac{d r_{b}}{d t}$ is of order of the disc gas drift velocity $v_{d r}$ near the secondary orbit. In general,

$$
v_{d r}=-\frac{3}{\Sigma r^{1 / 2}} \frac{\partial}{\partial r}\left(r^{1 / 2} \nu \Sigma\right),
$$

In the case of a massive secondary $\left(m \gg M_{d}\right)$, the second term in the round brackets can be neglected.

Prior to the moment $t=t_{0}$, we set $\frac{\partial \Sigma}{\partial t}=0$ into eq. (41). In this steady state case we have

$$
\begin{gathered}
\eta_{0} \equiv \nu_{0} \Sigma_{0}=\frac{\dot{M}}{3 \pi} \\
\Sigma_{0}=(3 k)^{-\frac{1}{1+a}}\left(\frac{\dot{M}}{\pi}\right)^{\frac{1}{1+a}} r^{-\frac{b}{1+a}}
\end{gathered}
$$

(C) RAS, MNRAS 000, 132 


$$
\begin{gathered}
v_{r 0}=-\frac{1}{2}(3 k)^{\frac{1}{1+a}}\left(\frac{\dot{M}}{\pi}\right)^{\frac{a}{1+a}} r^{\frac{b-a-1}{1+a}} \\
t_{\nu 0}=\frac{r}{\left|v_{r 0}\right|}=2(3 k)^{-\frac{1}{1+a}}\left(\frac{\dot{M}}{\pi}\right)^{-\frac{a}{1+a}} r^{\frac{c}{1+a}},
\end{gathered}
$$

where

$$
c=2(a+1)-b .
$$

We assume below that $c>0$.

The presence of the secondary in the disc significantly influences the structure of the inner part of the disc. Here we describe this influence in the most crude approximation ( a more accurate approach is discussed in the next subsection). The main effect of the secondary is to slow down the radial motion in the disc: $v_{r} \ll v_{r 0}$. To describe this effect quantitatively we divide the disc by two regions: 1) the outer region $r>r_{\text {inf }} \gg r_{b}$, where the equations (44-47) are approximately valid, 2) the inner region $r_{b}<r<r_{\text {inf }}$, where the drift velocity (43) is small compared to the stationary value (46). These regions are separated by the 'influence' radius $r_{\text {infl }}(t)$, and the dependence of $r_{i n f l}$ on time is calculated below. When $v_{r} \approx 0$, eq. (43) gives

$$
\Sigma(r, t)=C(t) r^{-\frac{b+1 / 2}{a+1}}
$$

The functions $C(t)$, and $r_{\text {infl }}(t)$ are calculated using the laws of conservation of mass and angular momentum. The eq. of mass conservation has a form

$$
\int_{0}^{r_{i n f l(t)}} d r\left(\frac{\partial}{\partial t} \Sigma r\right)=\frac{\dot{M}}{2 \pi}
$$

and the eq. of conservation of angular momentum has a form

$$
\int_{0}^{r_{i n f l(t)}} d r\left(\frac{\partial}{\partial t} \Sigma r^{3} \Omega\right)=\left\{\frac{3}{2} r^{2} \Omega \nu \Sigma\right\}_{r \rightarrow 0} .
$$

In both equations we formally extend the lower limit of integration to $r=0$. In eq. (50) we neglect the small inflow of angular momentum from the outer (steady-state) region of the disc. After substitution of (47) into eqs. (49-50), we have

$$
\begin{gathered}
\dot{C} r_{\text {infl }}^{\frac{2 c-1}{2(a+1)}}=\left\{\frac{2 c-1}{a+1}\right\} \frac{\dot{M}}{4 \pi}, \\
\dot{C} r_{\text {infl }}^{\frac{2 c+a}{2(a+1)}}=\frac{3}{4}\left\{\frac{2 c+a}{a+1}\right\} k C^{a+1} .
\end{gathered}
$$


The solution of eqs. (51-52) up to unimportant constant of integration has the form

$$
\begin{gathered}
C=(3 k)^{-\frac{2 c-1}{2(a+1) c}}\left(\frac{\dot{M}}{\pi}\right)^{\frac{2 c+a}{2(a+1) c}}(\beta t)^{\frac{1}{2 c}} \\
r_{\text {infl }}=(3 k)^{\frac{1}{c}}\left(\frac{\dot{M}}{\pi}\right)^{\frac{a}{c}}\left(\frac{2 c+a}{2 c-1}\right)^{2}(\beta t)^{\frac{a+1}{c}},
\end{gathered}
$$

where

$$
\beta=\left(\frac{c(2 c+a)}{2(a+1)}\right)\left(\frac{2 c+a}{2 c-1}\right)^{-\frac{2 c+a}{(a+1)}} .
$$

Prior to the moment $t_{0}$ the surface density in the disc is given by the steady-state solution (45), and after that moment, the surface density should be described by the solution (48, 53). To reconcile these two solutions we set

$$
t_{0}=\frac{t_{\nu 0}\left(r_{0}\right)}{2 \beta}
$$

where $t_{\nu 0}\left(r_{0}\right)=t_{\nu 0}\left(r=r_{0}\right)$. In this case eqs. (48), (53) can be written as

$$
\Sigma=\Sigma_{0}\left(r_{0}\right)\left(t / t_{0}\right)^{\frac{1}{2 c}}\left(r / r_{0}\right)^{-\frac{b+1 / 2}{a+1}}
$$

and for the viscosity distribution we have

$$
\eta=\frac{\dot{M}}{3 \pi}\left(t / t_{0}\right)^{\frac{a+1}{2 c}}\left(r / r_{0}\right)^{-1 / 2},
$$

where $\eta=\nu \Sigma$. The expressions (55-56) are valid as long as the local viscous time $t_{\nu}=\frac{r^{2}}{\nu}$ doesn't exceed the characteristic dynamical time $t_{d}=\frac{\Sigma}{\Sigma} \sim t$. We have

$$
t_{\nu} / t \sim\left(r / r_{i n f l}\right)^{\frac{2 c+a}{2(a+1)}}
$$

so our approximation breaks down at $r \sim r_{\text {infl }}$. Thus, to describe the disc structure at $r \sim r_{i n f l}$ we need a more accurate approach (see next subsection).

Now we are able to calculate the evolution of the binary orbit. First, let us calculate the secondary radial velocity $\frac{d r_{b}}{d t}$. For that we use eq. (42), and then neglecting the second term in the brackets we obtain

$$
\frac{d r_{b}}{d t}=-\frac{6 \pi r_{b} \eta\left(r_{b}, t\right)}{m}
$$

\footnotetext{
*** In the case of a relatively massive secondary, the inner edge of the disc is determined by a few outer Lindbland resonances, and may be 2-3 times larger than the secondary orbital radius (e.g. Artymowicz \& Lubow 1994). However we can use eq. (58) to calculate the radial velocity. This is connected with fact that the angular momentum outflow does not depend on the distance at all, see eq. (50).
}

(C) RAS, MNRAS 000, 132 
and with help of eq. (56) eq. (57) can be rewritten in dimensionless form

$$
\frac{d y_{b}}{d \tau}=-\frac{S}{2 \beta} \tau^{\frac{a+1}{2 c}},
$$

where $\tau=t / t_{0}, y_{b}=\left(r_{b} / r_{0}\right)^{1 / 2}$, and dimensionless parameter

$$
S=\frac{M_{d 0}}{m}<1
$$

here $M_{d o}=\dot{M} t_{\nu 0}\left(r_{0}\right)$. The integration of eq. (59) gives

$$
r_{b}=\left(1-\gamma S\left(\tau^{\frac{5 c+b}{4 c}}-1\right)\right)^{2} r_{0},
$$

where $\gamma=\frac{2 c}{\beta(5 c+b)} \sim 1$. The characteristic time-scale of the evolution of the secondary orbit $t_{e v}$ can be found from the condition $r_{b}\left(t_{e v}\right)=0$ :

$$
t_{e v}=(\gamma S)^{-\frac{4 c}{5 c+b}} t_{0} .
$$

It is instructive to compare the expression (60) with the Syer and Clarke result:

$$
r_{b} \approx\left(1-\beta_{S C} S^{\frac{1}{2+a}} \tau\right)^{\frac{2+a}{c}} r_{0},
$$

where the dimensionless coefficient $\beta_{S C}=2^{-\frac{1+a}{2+a}} \frac{c}{\beta(2+a)} \sim 1$. It can be seen that for the typical values of the parameters $a, b$ the evolution equation (62) underestimates the evolution time in comparison with equation (60).

The dimensionless velocity (59) taken at the time $t_{e v}$ can be estimated as:

$$
\left|\frac{d y_{b}}{d \tau}\right| \sim S^{\frac{4 c}{5 c+b}}<1 .
$$

This inequality implies that we can treat the change of binary orbit as a slow effect with respect to the change of the disc configuration when $t<t_{e v}$. On the other hand, let us look at the ratio of the disc mass contained inside the region influenced by the secondary $\left(r_{b}<r<r_{\text {infl }}\right)$ to the mass of the secondary. It can be easily seen that this ratio is small at the time $t \sim t_{e v}$ :

$$
M_{d}=2 \pi \int_{r_{b}\left(t_{e v}\right)}^{r_{i n f l}\left(t_{e v}\right)} d r r \Sigma\left(t_{e v}, r\right) \sim S^{\frac{2(a+1)}{5 c+b}} m<m,
$$

and this means we can use eq. (58) as a boundary condition to our problem. It is useful to express the evolutionary time $t_{e v}$ in terms of the accretion time $t_{a c c}=\frac{m}{\dot{M}}$. From eq. (61) we obtain

$$
t_{e v}=(\gamma S)^{\frac{2(a+1)}{5 c+b}} t_{a c c}<t_{a c c} .
$$

Say, for $a=2 / 3, b=1$, we have

$$
t_{e v}=(\gamma S)^{5 / 19} t_{a c c}
$$

(C) RAS, MNRAS 000, 12 
and for $a=3 / 7, b=15 / 14$, we have

$$
t_{e v}=(\gamma S)^{2 / 7} t_{a c c}
$$

Finally let us estimate the luminosity of the disc. From the law of energy conservation it follows that the total luminosity of the disc $L$ must be equal to the change of the secondary energy per unit of time:

$$
L=-\frac{1}{2} \frac{G m M}{r_{b}^{2}} \frac{d r_{b}}{d t}=\frac{G M \dot{M}}{r_{b}(t)}\left(\frac{r_{0}}{r_{b}(t)}\right)^{1 / 2}(\tau)^{\frac{a+1}{2 c}},
$$

where $r_{b}(t)$ is given by eq. (60). When the separation distance $r_{b}$ is much larger than the gravitational radius of the primary, the "initial" value of luminosity (taken at the moment $\left.t=t_{0}\right)$ is much smaller than the "standard" value of luminosity of the steady state disc $L_{s t}$

$$
L_{s t} \sim \frac{\dot{M} M}{r_{g}} \approx 10^{44} \dot{M}_{*} M_{8} \frac{\mathrm{erg}}{\mathrm{s}} .
$$

However, the luminosity grows with time due to evolution of inner part of the disc and the secondary orbit.

\subsection{Self-similar solutions}

Now we consider in detail the formal case of infinitely small binary separation distance $\left(r_{b} \approx 0\right)$. As we will see, in this case simple self-similar solutions of eq. (41) can be found

**. These solutions are close to the exact solutions for the disc structure outside the binary orbit, and they allow us to find the disc structure at the intermediate scales $r \sim r_{\text {infl }}$.

When considering the case $r_{b}=0$, we must specify the boundary conditions at $r=0$ and at infinity. We assume that there is no radial velocity at $r=0: v_{r}(r=0)=0$, and the disc structure tends to the steady solution with increase of $r$. We look for a solution of eq. (41) in the form

$$
\begin{aligned}
& \sigma=t^{-m} F(\xi), \\
& \xi=D^{-1} r t^{-n},
\end{aligned}
$$

where we use the surface number density $\sigma=\Sigma / m_{p}\left(m_{p}\right.$ is the proton mass), and the constant $D$ is chosen to make the similarity variable (71) dimensionless. The powers $n, m$

*** Our approach is similar to the Lyubarskii and Shakura 1987 study of non-stationary disc accretion, and to Pringle's 1991 calculation of evolution of external circumbinary disc of a given mass.

(C) RAS, MNRAS 000, 132 
are specified by the condition that eq. (41), as well as the accretion rate

$$
\frac{\dot{m}}{2 \pi} \equiv \frac{\dot{M}}{2 \pi m_{p}}=3 r^{1 / 2} \frac{\partial}{\partial r}\left(r^{1 / 2} \nu \sigma\right),
$$

are expressed in terms of the variable $\xi$ only. Substituting eqs. (70) and (71) into eqs. (41) and (72), we obtain

$$
n=\frac{1+a}{c}, \quad m=\frac{b}{c} .
$$

Equation (41) takes the form

$$
(3 \tilde{k}) D^{b-2} \frac{d}{d \xi} \xi^{1 / 2} \frac{d}{d \xi} \xi^{1 / 2+b} F^{1+a}+\xi\left(n \xi \frac{d}{d \xi} F+m F\right)=0,
$$

with

$$
D=(3 \tilde{k})^{\frac{1}{c}}\left(\frac{\dot{m}}{\pi}\right)^{\frac{a}{c}},
$$

where $\tilde{k}=m_{p}^{a} k$. The solution of eq. (74) should be separately analyzed for the general situation $a \neq 0$, and for the degenerate case $a=0$.

In the case $a=0$ we have $(3 \tilde{k}) D^{b-2}=1$, Eq. (74) is linear, and can be easily solved. The general solution is

$$
F=\xi^{-b}\left(C_{1}+C_{2} \int_{0}^{\xi^{-1 / 2}} d x e^{-\frac{x^{-2(2-b)}}{(2-b)^{2}}}\right) .
$$

The first term in the brackets represents the stationary solution (45) provided $C_{1}=1$. We have

$$
F \approx C_{2} \xi^{-b-1 / 2},
$$

at $\xi \rightarrow 0$, and the comparison of eqs. (70,71), (77), and (48), (53) gives $C_{2}=\beta$.

In the case $a \neq 0$, we can eliminate dimensional factor in the eq. (74) by redefining of the function $F$

$$
F=(3 \tilde{k})^{-\frac{1}{a}} D^{\frac{2-b}{a}} \tilde{F} .
$$

Substituting (73) (77) into (74), we obtain:

$$
\frac{d}{d \xi} \xi^{1 / 2} \frac{d}{d \xi} \xi^{1 / 2+b} \tilde{F}^{1+a}+\frac{\xi}{c}\left(b \tilde{F}+(1+a) \xi \frac{d}{d \xi} \tilde{F}\right)=0 .
$$

In the limit $\xi \gg 1$ the solution of eq. (79) is close to the stationary solution

$$
\tilde{F}_{0}=\xi^{-\frac{b}{1+a}},
$$

and in the opposite limit $\xi \ll 1$ we have

$$
\tilde{F}=C \xi^{-\frac{b+1 / 2}{1+a}} .
$$

(C) RAS, MNRAS 000, 12 


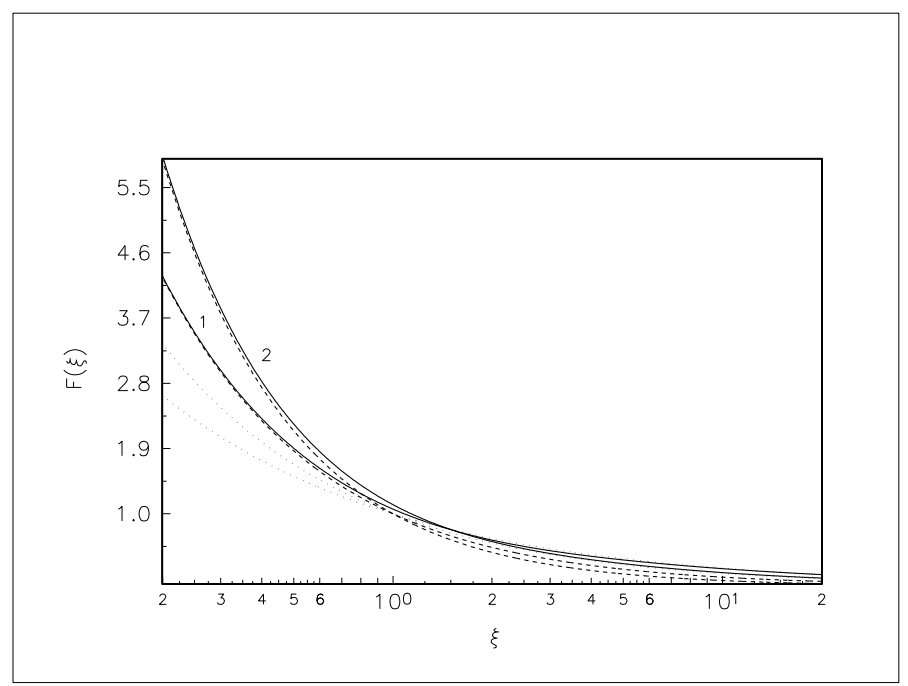

Figure 4. We plot the numerical solution of eq. (79) $F(\xi)$ as a function of the similarity variable $\xi$ (solid curves). Curve 1 corresponds to the case $a=2 / 3, b=1$ (Thompson opacity dominates), and the curve 2 corresponds to the case $a=3 / 7$, $b=15 / 14$ (the opacity is determined by the free-free processes). The dashed lines represent the analytical asymptotic of that solutions at $\xi \rightarrow 0$ (see eq. (81)), and the dotted lines represent the stationary solutions of eq. (79) (see eq. (80))

It is evident from eqs. $(70,71,81)$ that the asymptotic $(81)$ corresponds to the approximate solution $(48,53)$ if we set again $C=\beta$. Thus eqs. $(45)$ and $(48,53)$ give asymptotic limits to the self-similar solution of eq. (41). The intermediate scale $\xi \sim 1$ separates the asymptotic $(80,81)$, and the corresponding radius $r_{*}(\xi=1, t)$ is of order of $r_{\text {infl }}$.

The numerical solution to eq. (79) is presented in Fig. 4. One can see from this Fig., that the solution of this equation approximated with high accuracy by the expression (81) at $\xi<1$, and by the expression (80) at $\xi>1$. We will use this fact below comparing our analytical estimates with the results of the numerical computations.

\subsection{Numerical model}

We solve numerically equation (41), and compare the results of our calculations with analytical estimates. For numerical work we rewrite eq. (41) in terms of new radial coordinate $y=\left(r / r_{0}\right)^{1 / 2}$, dimensionless time $\tau=t / t_{0}$, and dimensionless surface density $\tilde{\Sigma}=\Sigma / \Sigma_{0}\left(r_{0}\right)$ :

$$
\frac{\partial \tilde{\Sigma}}{\partial \tau}=\frac{1}{4 \beta} y^{-3} \frac{\partial^{2}}{\partial y^{2}}\left(y^{1+2 b} \tilde{\Sigma}^{1+a}\right) .
$$

To solve this equation, we must specify the initial and boundary conditions. We assume that the disc surface density is equal to its steady state value (45) at some point $y_{\text {out }} \gg 1$, and we take $y_{\text {out }}=10^{2}$ in our calculations. Since we are interested in the effect of evolution of the disc and the secondary orbit, the detailed description of the angular momentum exchange 
between the secondary and the disc is unimportant for us, and to obtain a boundary condition at $y=y_{b}$ we substitute the expression for the disc gas radial velocity (43) in eq. (42) and use the resulting equation as the boundary condition to our problem. The eq. (43) allows us to calculate the change of the secondary orbit. The initial surface density distribution $\Sigma(t=0, y)$ is determined by eq. (45) in the range $2<y<y_{\text {out }}$, and we use the appropriate polynomial function in the range $1<y<2$ to match the steady state distribution and the inner boundary condition at the moment $t=0$. The detailed form of this function is unimportant for us. The position of the secondary $y_{b}=\left(r_{b} / r_{0}\right)^{1 / 2}$ is changing with time, and in our numerical scheme it is convenient to use another radial coordinate $\tilde{y}$ which is stretching with change of the inner boundary with respect to coordinate $r$ :

$$
\tilde{y}=\frac{\left(y_{\text {out }}-1\right) y+y_{\text {out }}\left(1-y_{b}(t)\right)}{y_{\text {out }}-y_{b}(t)},
$$

and $\tilde{y}\left(y_{b}(t)\right)=1, \tilde{y}\left(y_{\text {out }}\right)=y_{\text {out }}$. We use uniform mesh in coordinates $(\tau, \tilde{y})$ with $10^{4}$ grid elements in $\tilde{y}$-direction, and employ a standard implicit scheme. To check the relaxation of numerical solution to the self-similar asymptote, we integrate eq. (82) with respect to time up to the time $t_{\text {out }}=2 t_{e v}$. When the orbital distance approaches the value $y_{\text {in }}=10^{-2}$ we "turn off" the radial evolution of the secondary.

The results of numerical calculations are presented at Figs. (5-7). In Figs. 5,6 we show the calculated density profile taken in different moments of time, and for different parameters of our model. One can see from these Figs. that the surface density of the disc is well approximated by the analytical expressions (45), (55). In Fig. 7 we show the dependence of the secondary orbital distance on time. The analytical curves give again very good approximation to the numerical results.

\section{ASTROPHYSICAL CONSEQUENCES AND CONCLUSIONS}

Now let us summarize the main results of our paper. We considered the interaction between a massive binary system and an accretion disc, paying special attention to the case when the secondary mass is larger than the disc mass inside the binary orbit. We showed that the joint evolution of the binary and disc could be divided into several successive stages. When the binary orbital plane is inclined with respect to plane of the disc, the gravitational field of the binary causes non-axisymmetric "twisted" distortions of an initially planar disc,

(C) RAS, MNRAS 000, 132 


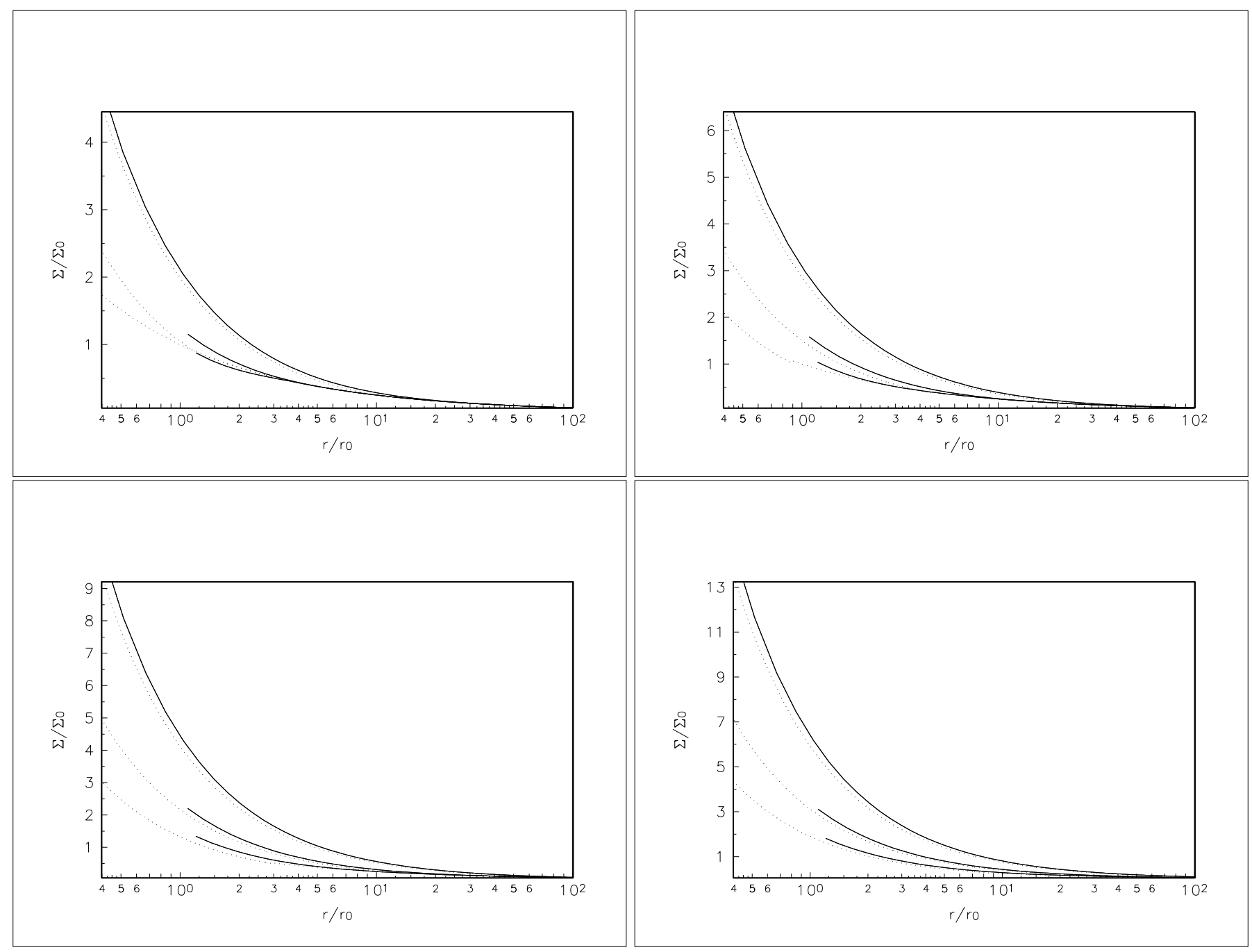

Figure 5. The dimensionless surface density $\Sigma / \Sigma_{0}\left(r_{0}\right)$ is plotted against dimensionless radius $r / r_{0}$. The parameters $a=2 / 3$ and $b=1$ correspond to the case of Thompson opacity "domination". The different plots correspond to the different values of the parameter $S$ : a) top left plot $-S=10^{-1}$, b) top right plot- $S=10^{-2}$, c) bottom left plot $-S=10^{-3}$, d) bottom right plot$S=10^{-4}$. The solid curves, in order of increasing of inner boundary, are made at times $t=10^{-2} t_{e v}, t=10^{-1} t_{e v}$ and $t=2 t_{e v}$. The dotted curves are determined by eqs. $(45,55)$. In asymptotics the solutions have power-law form: $\Sigma(r \rightarrow \infty) \sim r^{-3 / 5}$, and $\Sigma(r \rightarrow 0) \sim r^{-9 / 10}$.

and the inner part of the disc lies in the orbital plane as a result of the evolution of these distortions. Since the orientation of outer part of the disc is controlled by the disc formation processes, after the alignment of the inner part of the disc a quasi-stationary twisted disc is formed. We calculate the radial dependence of the inclination angle of the twisted disc, and find the dependence of the characteristic alignment scale on viscosity and mass ratio of the binary components. It is shown that in the low-viscosity limit the alignment scale does not depend on the value of the viscosity parameter $\alpha$.

Assuming that the inner part of the disc lies in the binary orbital plane, rotates in the 


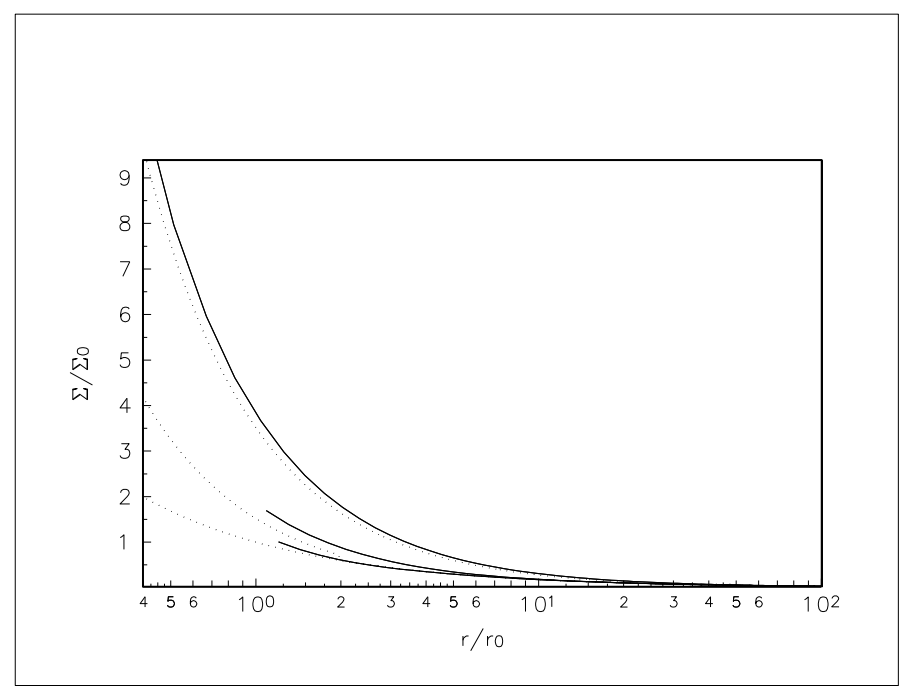

Figure 6. The same as the plot b) of Fig. 5, but now $a=3 / 7, b=15 / 14$ (the opacity is determined by free-free processes). The general behavior of the curves is similar to the case b), but the asymptotical slope is slightly steeper: $\Sigma(r \rightarrow \infty) \sim r^{-3 / 4}$, and $\Sigma(r \rightarrow 0) \sim r^{-11 / 10}$
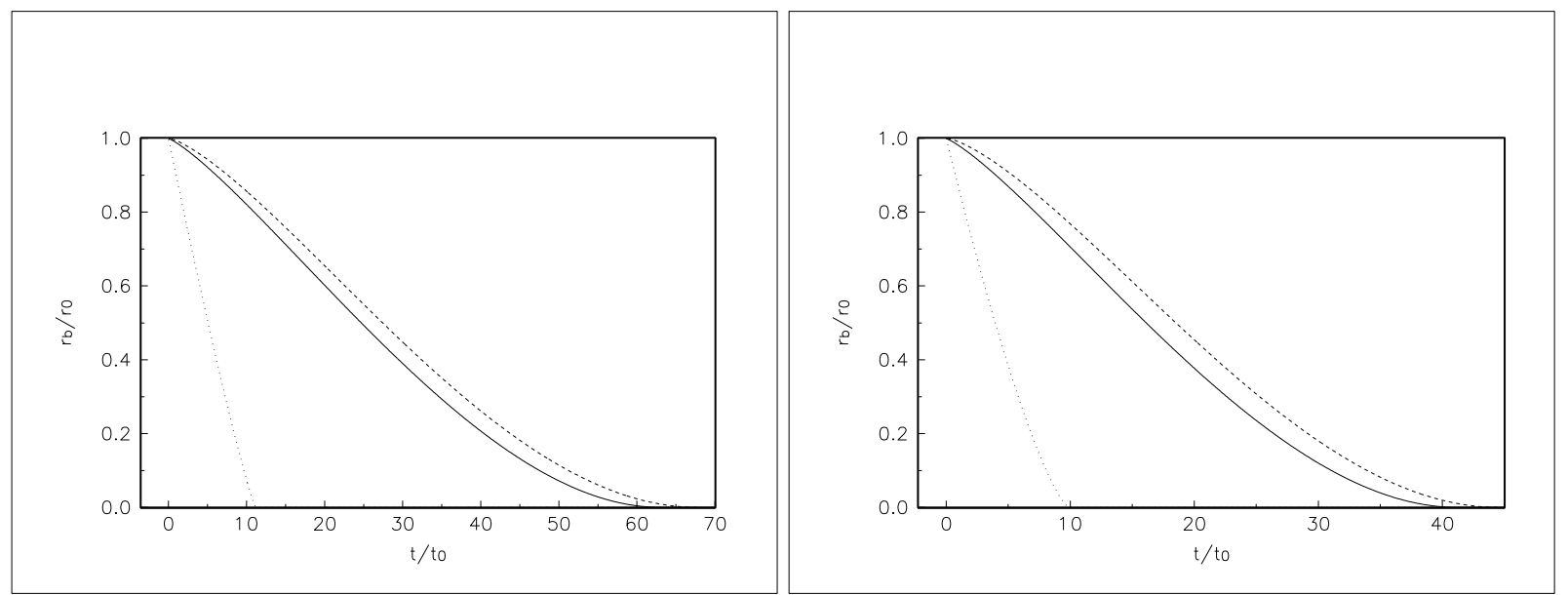

Figure 7. The dimensionless binary separation distance $r_{b} / r_{0}$ is plotted against the time $t / t_{0}$. The parameters of the left plot are the same as in the plot b) of Fig. 5, and the parameters of the right plot are the same as in Fig. 6. The dashed curves are analytical expressions given by eq. (60), and the dotted curves represent Syer and Clarke result (62)

same sense as the binary, we find the radial evolution of the disc structure and the evolution of the binary separation distance. In such a situation the disc gas cannot drift through the binary orbit, and is accumulated in some region outside the binary orbit. The structure of the disc in this region is close to the self-similar solution of the diffusion equation describing the viscous evolution of the disc surface density. The back reaction of the circumbinary disc pushes the secondary inwards towards the primary. We calculate analytically the dependence of the binary separation distance on time, and compare with results of numerical calculations. We find that the characteristic time scale of this evolution is determined by the ratio $S$ of 
the disc mass inside the initial binary orbit and the secondary mass $m$. The evolution time scale is always smaller than the "accretion" time scale $t_{a c c}=\frac{M}{M}$.

In the case of a supermassive black hole binary, evolution of the binary separation distance is also caused by the emission of gravitational waves. To estimate the relative significance of this effect, let us compare the total disc luminosity with orbital energy loss $\dot{E}_{g w}$ due to emission of gravitational waves (Peters \& Mathews 1963)

$$
\dot{E}_{g w}=\frac{1}{5} L_{*} q^{2}\left(\frac{r_{g}}{r_{b}}\right)^{5}
$$

where the "gravitational emission factor" $L_{*}=\frac{c^{5}}{G} \approx 3.6 \times 10^{59} \mathrm{erg} / \mathrm{s}$. Comparing eqs. (69), and (84), we find that the interaction with the accretion disc is more important at scales larger than the characteristic "gravitational" scale $r_{g w}$ :

$$
r>r_{g w} \approx 6 \cdot 10^{2}\left(\frac{q}{10^{-2}}\right)^{1 / 2}\left(M_{8} \dot{M}_{*}\right)^{-1 / 4} r_{g} .
$$

As we have already discussed, the evolution of a supermassive black hole binary due to dynamical friction with the stars of the central stellar cluster can be unimportant at scales $<1 p c$, and therefore the secondary-disc interaction may provide the only possibility to pass through the "intermediate" scales $r_{g w}<r<1 p c$. This fact may be critically important for formation of powerful sources of gravitational radiation due to coalescence of supermassive black holes.

One uncertainty in our calculation seems to be connected with eccentricity evolution. In the course of binary evolution due to dynamical friction with the stars, the eccentricity seems not to grow significantly (see, e. g. Polnarev \& Rees 1994 for analytic work and Quinlan \& Hernquist 1997 for N-body simulations). However, the binary-disc interaction may induce some eccentricity (e.g. Artymowicz, 1992, Lin \& Papaloizou, 1993). As we have mentioned, gravitational wave emission is much more effective for eccentric binaries, and a significant eccentricity can shorten the evolutionary time scale.

$* * *$ In the expression (84) we set the orbital eccentricity $e=0$. For $e \sim 1$ the emission of gravitational waves is much more effective (Peters \& Mathews 1963).

(C) RAS, MNRAS 000, 132 


\section{ACKNOWLEDGMENTS}

We thank N. I. Shakura and C. Terquem for useful comments. We are also grateful to the referee for important remarks. PBI and JCBP thank the Isaac Newton Institute for Mathematical Sciences for hospitality. This work was supported in part by RFBR grants N 96-02-16689a, 97-02-16975 and by Danish Research Foundation through its establishment of the Theoretical Astrophysics Center.

\section{REFERENCES}

Artymowicz P., 1992, PASP, 104, 769A

Artymowicz P., 1994, ApJ, 423, 581

Artymowicz P., Lubow S. H., 1994, ApJ, 421, 651

Bahcall J. H., Wolf R. A., 1976, ApJ, 209, 214

Begelman M. C., Blandford R. D., and Rees M. J., 1980, Nature, 287, 307-309

Coleman C. S., Kumar S., 1993, MNRAS, 260, 323

Demianski M., Ivanov P. B., 1997, A\&A 324, 829

Goldreich P., Tremaine S., 1980, ApJ, 241, 425

Ivanov P. B., Illarionov A. F., 1997, MNRAS, 285, 394

Ivanov P. B., Igumenshchev I. V., and Novikov I. D., 1998, ApJ, 507, 131

Illarionov A. F., Romanova M. M., 1988, SvA, 32, 274

Jensen E. L., Mathieu R. D., and Fuller G. A., 1996, ApJ, 458, 312

Jensen E. L., Mathieu R. D., 1997, AJ, 114, 301

Kumar S., 1987, MNRAS, 225, 823

Kumar S., 1990, MNRAS, 245, 670

Lehto N. J., Valtonen M. J., 1996, ApJ, 460, 207

Lightman A. P., Eardley D. M., 1974, ApJ, 187, L1

Lin D.N.C., Papaloizou J. C. B., 1979, MNRAS, 186, 799

Lin D.N.C., Papaloizou J. C. B., 1986, ApJ, 309, 846

Lin D.N.C., Papaloizou J. C. B., 1993, In: Protostars and planets III (A93-42937 17-90), p. 749-8, University of Arizona press.

Lyubarskii Y.E., Shakura N.I., 1987, SvA, 13, 386

Papaloizou J.C.B., Lin D.N.C., 1995, ApJ, 438, 841

Papaloizou J.C.B., Pringle J.E., 1983, MNRAS, 202, 1181

Papaloizou J.C.B., Terquem C., Lin D.N.C., 1998, Ap.J., 497, 212

Peters P. C., Mathews J., 1963, Phys. Rev., 131, 435

Petterson J. A., 1977, ApJ, 214, 550

Polnarev A. G., Rees M. J., 1994, A\&A, 283, 301

Pringle J. E., 1991, MNRAS, 248, 754

Quinlan C.D., 1996, NewA., 1, 35

Quinlan C.D., Hernquist L., 1997, NewA., 2, 533

Rees M. J., 1978, Nature, 275, 516

(c) RAS, MNRAS 000, 132 
Shakura N. I., Sunyaev R. A., 1973, A\&A, 24, 335

Spitzer L., Saslaw W., 1966, Ap. J., 143, 400

Scheuer P.A.G., Feiler R., 1996, MNRAS, 282, 291

Syer D., Clarke C. J., 1995, MNRAS, 277, 758

Vokrouhlicky D., Karas V., 1998, MNRAS, 293 L1

Ward W. R., Icarus, 1996, 126, 261

Young P. J., 1977, ApJ, 215, 36

This paper has been produced using the Royal Astronomical Society/Blackwell Science IATEX style file. 